\title{
A Digital Photographic Technique for Knee Range of Motion Measurement: Performance in a Total Knee Arthroplasty Clinical Population
}

\author{
Michael Murphy ${ }^{1}$, Julie Hides ${ }^{2}$, Trevor Russell ${ }^{3}$ \\ ${ }^{1}$ Mater Medical Research Institute, Brisbane, Australia; ${ }^{2}$ Faculty of Health Sciences, Australian Catholic University, Brisbane, Aus- \\ tralia; ${ }^{3}$ School of Health and Rehabilitation Science, University of Queensland, Brisbane, Australia. \\ Email: michael.murphy@mater.org.au
}

Received January $15^{\text {th }}, 2013$; revised March $1^{\text {st }}, 2013$; accepted March $18^{\text {th }}, 2013$

\begin{abstract}
Background: Knee range of motion is an essential outcome measure following total knee arthroplasty and it is important to measure this outcome with a simple and accurate tool. Measurement from digital photographs could provide a superior clinical alternative to the common goniometer as devices for capturing digital photographs are becoming more accessible, measurement accuracy is assisted with the associated software and images can be saved and stored for later reference. This ability to store images with measurements could be particularly useful in research involving knee range of motion. This study evaluated the validity and reliability of a simple photographic measurement technique to measure knee flexion and extension of patients following knee arthroplasty. Methods: Knee flexion and extension of 38 subjects who had undergone total knee arthroplasty were assessed at the one year post-operative review with the digital technique and a universal goniometer as the criterion reference. Digital measurements were repeated one month later by the first assessor (intra-tester reliability) and another independent assessor (inter-tester reliability). Results: Validity: the digital technique was found to have a high level of agreement with the universal goniometer measurements (all MAD < $2.35^{\circ}$ ). Reliability: small differences were observed in intra and inter-tester measurements for both flexion and extension with almost perfect Intra-Class Correlations (Intra-tester; flexion 0.99, extension 0.96; inter-tester; flexion 0.97, extension 0.93). Conclusion: Measurement of knee range of motion from digital photographs can be used in routine clinical total knee arthroplasty follow-up instead of, or interchangeably with the universal goniometer.
\end{abstract}

Keywords: Knee Range of Motion; Arthroplasty; Digital Photographic Goniometry

\section{Introduction}

A major goal of total knee arthroplasty (TKA) is to achieve sufficient knee range of motion (ROM) for routine daily activities [1]. Accurate, reliable measurement of knee ROM is therefore essential for determining the success of the procedure as well as guiding decision making for progression of rehabilitation or further intervention such as manipulation. Moreover it is particularly critical in studies evaluating interventions designed to improve ROM such as with the recent emergence of "high-flex" prostheses [2,3].

The lateral radiograph is generally accepted as the gold standard for knee ROM measurement [4], however for routine post-operative TKA review, radiographs of the knee in both extreme flexion and extension are not clinically indicated. Clinicians therefore, tend to use either visual estimation which is useful for the experienced observer but not reliable [5], or more commonly goni- ometry. The most common goniometer, the universal goniometer (UG) is simple, and inexpensive [6] and has demonstrated validity when compared to the lateral radiograph (ICC $=0.98-0.99)$ and excellent reliability (ICC $=0.99)$ [4]. Furthermore it is being increasingly used as the criterion standard in research [4,7-10]. UG measurements however are potentially confounded by variability in training or experience of the measurer $[3,5]$, the positioning of the patient [11] and accuracy of localisation of landmarks particularly with obese patients $[5,6$, 12 ], and these variables are not recorded with the simple numerical value output of the UG measurement.

Measurement of knee ROM from digital photographs or Digital Photographic Goniometry (DPG) has recently emerged as an option for ROM measurement [12-15] and provides visual detail on many of the variables such as measurement positioning and limb shape not apparent with UG recordings. Compact digital cameras are readily accessible particularly as they are now incorporated in 
Performance in a Total Knee Arthroplasty Clinical Population

most modern mobile phones. The image analysis software required for ROM analysis can be installed onto smart phones for instant calculation of ROM. The software allows the assessor to place points directly on the image at the usual goniometric landmarks (e.g. greater trochanter and lateral malleolus) which are usually beyond the reach of the standard UG arms. This reduces the risk of error with inaccurate goniometer arm placement [15]. As an additional benefit, images can be securely stored allowing clinicians or researchers to review and check measurements at a later date $[12,14]$. Technology of this nature has been utilised in telemedicine [8], and has potential for use in the operating theatre where a UG poses an infection risk [6].

Several studies have reported excellent inter-tester and intra-tester reliability with DPG of knee flexion and extension and established its validity against radiographs [12] and the UG $[8,14]$. However, typical of most ROM assessment studies which are conducted in healthy populations [7], no studies have evaluated the knees of subjects who had undergone TKA. Furthermore previous studies have involved a variety of photographic protocols and research methodologies of varying rigour. The aim of this study was to investigate the validity and reliability of a simple photographic method used in the clinical setting to measure knee flexion and extension after TKA.

\section{Materials and Methods}

\subsection{Subjects}

This study assessed participants in a randomised controlled trial evaluating the effect of femoral implant position on knee flexion following TKA with the Profix Total Knee System (Smith \& Nephew, Memphis, TN), (Australian New Zealand Clinical Trials Registry, 12606000325505). The study has institutional ethical approval and informed consent was obtained from each participant. Data from 38 participants who attended for routine one year post-operative review were used in the study. The mean age of participants was 71.0 years (standard deviation 9.5), twenty-three were female and mean Body Mass Index was 29.3 (standard deviation 5.8).

\subsection{Protocol}

Passive knee flexion and extension were measured using a standard $30 \mathrm{~cm} \mathrm{UG}$ as the criterion measure and photographs were taken with a digital camera (Canon, Powershot A620 at an image resolution of $1600 \times 1200$ pixels). Knee extension was measured with the subject supine in neutral hip rotation and with their ankle supported to prevent lower leg contact with the bed. Knee flexion was measured with the hip flexed to approximately ninety degrees and the knee passively flexed with gravity as previously described in TKA literature [16,17]. The landmarks for knee ROM assessment described by Norkin and White [18] were used for both UG and DPG measurements. The UG axis was placed at the lateral epicondyle; the arms aligned with the femur with the greater trochanter as the reference, and the fibula with the lateral malleolus as the reference. Immediately following the UG measurement, whilst knee position was maintained, the digital photograph of the subject's lower limb was taken. The aim of the study was to investigate the utility of a simple photographic method, therefore camera settings were set to automatic and the image taken with the camera visually aligned with the lateral epicondyle perpendicular to the lower limb. This was deemed sufficient to ensure the camera was positioned within a previously demonstrated allowable margin of $5^{\circ}$ which minimises the risk of parallax error [13].

Images were transferred via the camera's standard memory card (SmartMedia card drive (SanDisk (Sunnyvale, CA, USA)), identified with a unique study number and saved as a computer file. ROM was measured with a 2-dimensional kinematic software analysis application that has been adapted from a measurement suite (Version 2.4) which ships with the $\mathrm{eHAB}^{\circledR}$ telerehabilitation system (NeoRehab, Brisbane). This software has been specifically developed to measure joint angles and accounts for variables such as camera lens and parallax errors. With this software the assessor selects the landmarks with the computer mouse and the software automatically connects the landmarks with lines superimposed on the image and displays the angle (Figure 1). The assessor can then assess the orientation of the lines and make easy quick adjustments by dragging the landmark points to ensure the lines align with the femur and fibula. This reduces the risk of inaccuracy of landmark positioning

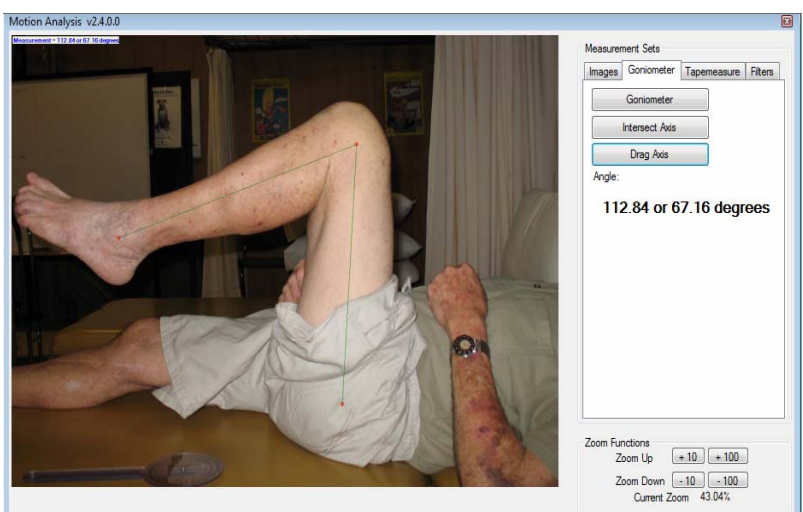

Figure 1. Screen capture of the 2-dimensional kinematic software analysis application $\left(\mathrm{eHAB}^{\circledR}\right.$ telerehabilitation system (Version 2.4, NeoRehab, Brisbane)) showing placement of the bony landmarks; greater trochanter, lateral malleolus and lateral epicondyle. 
which are palpated with UG measures. The image, together with the superimposed lines and joint angle, can be saved on the photographic image file for future reference, however for this study software graphics were not saved to ensure assessors were blinded to previous and each other's measurements.

Two physiotherapists (assessors 1 \& 2) experienced in musculoskeletal assessment participated in the study. Assessor 1 had taken all UG assessments and photographs for the subjects in the RCT. UG measurements were recorded on the day of each participant's one year post-operative clinic review and photographic images were saved onto a dedicated study computer. Assessor 1 performed the DPG measurements of all one year review photographs two months or more after the final participant's review. As subjects were recruited over a two year period, DPG measurements therefore ranged from two months to two years after the UG measurements, minimising the likelihood of recall of the UG values. One month following the initial DPG measurements assessor 1 repeated the DPG measurements for intra-tester reliability analysis and assessor 2 conducted DPG measurements for inter-tester reliability analysis.

\subsection{Statistical Analysis}

All data were analysed using the SPSS software package version 15.0 (SPSS Inc. Chicago, Illinois). To test the validity of the DPG in comparison to the UG the mean absolute difference (MAD) and Bland and Altman's $[19,20]$ "Limits of Agreement" (LOA) method of assessing agreement were chosen. This method assesses the strength of the agreement of the two measurement techniques. Plots of the difference in measurement for knee flexion and extension between the criterion and DPG techniques versus the average of these measurements were constructed and examined for heterogeneity as described by Bland and Altman [19]. To analyse inter and intra-rater reliability of the DPG measurements the MAD and Intra Class Correlation ICC $(2,1)$ were selected. An ICC between 0.61 and 0.80 represents substantial reproducibility and between 0.81 and 1.00 almost perfect reproducibility [21].

\section{Results}

The means and standard deviations of knee flexion and extension for the subjects using the DPG measurements were $106.5^{\circ}\left(10.0^{\circ}\right)$ and $6.5^{\circ}\left(6.0^{\circ}\right)$ respectively.

The results for the validity and reliability analyses of knee flexion and extension are detailed in Table 1.

\subsection{Validity}

The mean differences between the DPG and the UG for both knee flexion and extension were small and close to zero indicating minimal to no systematic measurement error. The MAD's for knee flexion and extension were also small (flexion $2.01^{\circ}$, extension $2.35^{\circ}$ ) indicating minimal random error. The range of the LOA was $10.58^{\circ}$ in flexion and $10.43^{\circ}$ in extension.

\subsection{Reliability}

\subsubsection{Intra-Tester Reliability of DPG Measurements} Excellent ICC's were observed when the same assessor measured knee flexion and extension $(0.99,0.96$ respectively), with knee flexion slightly stronger. MAD's and mean differences were small and close to zero with knee extension that were marginally less than knee flexion (MAD: $1.28^{\circ}$ and $1.35^{\circ}$ respectively, mean: $-0.05^{\circ}, 0.79^{\circ}$ respectively).

\subsubsection{Inter-Tester Reliability of DPG Measurements} Excellent ICC's were observed when the different assessors measured knee flexion and extension with flexion again slightly higher $(0.97,0.93$ respectively). MAD's

Table 1. Validity and reliability of Digital Photographic Goniometry. SD—standard deviation, MAD—mean absolute difference, ICC-Intra Class Correlation $(2,1)$. UG-universal goniometer. DPG-Digital Photographic Goniometry. Intra-tester reliability: $\mathrm{A1}^{1}$-assessor 1 , measurement 1 ; $\mathrm{A1}^{2}$-assessor 1 , measurement 2 . Inter-tester reliability: $\mathrm{A1}$-assessor 1 , A2-assessor 2.

\begin{tabular}{|c|c|c|c|c|c|c|}
\hline & & Mean difference (degrees) & $\mathrm{SD}$ (degrees) & MAD (degrees) & $\begin{array}{c}95 \% \text { Limits of } \\
\text { Agreement (degrees) }\end{array}$ & ICC \\
\hline \multirow{2}{*}{$\begin{array}{c}\text { Validity } \\
\text { UG-DPG }\left(\mathrm{A}^{1}\right)\end{array}$} & Flexion & -0.24 & 1.78 & 2.01 & $-5.53-5.05$ & - \\
\hline & Extension & -1.03 & 1.58 & 2.35 & $-6.24-4.19$ & - \\
\hline \multirow{2}{*}{$\begin{array}{l}\text { Intra-tester reliability } \\
\qquad \mathrm{A} 1^{1}-\mathrm{A} 1^{2}\end{array}$} & Flexion & 0.79 & 0.69 & 1.35 & - & 0.99 \\
\hline & Extension & -0.05 & 0.99 & 1.28 & - & 0.96 \\
\hline \multirow{2}{*}{$\begin{array}{l}\text { Inter-tester reliability } \\
\qquad \mathrm{A} 1-\mathrm{A} 2\end{array}$} & Flexion & 0.80 & 1.36 & 1.91 & - & 0.97 \\
\hline & Extension & 0.93 & 1.33 & 1.71 & - & 0.93 \\
\hline
\end{tabular}


Performance in a Total Knee Arthroplasty Clinical Population

and mean differences were slightly greater than intratester results but were similarly small and close to zero.

\section{Discussion}

Valid and reliable measurement of knee ROM is an essential element of assessment at post-operative TKA review. This study examined the clinimetric properties of measurement of knee flexion and extension of TKA patients at routine clinical reviews using a simple photographic method and found excellent criterion-related validity and intra and inter-tester reliability. In both knee flexion and extension there was no indication of systematic error and minimal random error as mean and mean absolute differences were very small and clinically insignificant. These results imply that clinicians can confidently use the DPG instead of, or interchangeably with the UG.

Several studies have evaluated knee ROM with DPG but with a variety of populations and methodologies ranging from procedures involving rigorous camera and tripod preparation $[6,8,14]$, to hand held digital cameras similar to that used in our study but with measurement taken from printed photographs [12]. Some studies, like ours, used software programs to assess ROM from digital photographs, most using goniometric landmarks $[8,14]$ and one used lines parallel to the anterior surfaces of the thigh and lower leg to reduce potential error from inaccurate landmark localisation [6]. Our technique involved minimal preparation using a hand held camera visually aligned with the knee axis and we found good agreement between DPG and UG measurements. Furthermore the range of the LOA in our study for both flexion $\left(-0.53^{\circ}\right.$ to $\left.5.05^{\circ}\right)$ and extension $\left(-6.24^{\circ}\right.$ to $\left.4.19^{\circ}\right)$ were within acceptable clinical limits.

Naylor et al. (2011) reported a range of LOA for three assessors between $14.1^{\circ}$ and $18.7^{\circ}$ for flexion and between $25.1^{\circ}$ and $31.3^{\circ}$ for extension with their "line of femur" technique. This technique involved using the lateral malleolus and knee joint centre as reference points and the estimated line along the long axis of the femur. That study used lateral radiographs as the criterion standard and as the radiographs did not capture the goniometric landmarks of lateral malleolus and greater trochanter, measurements were taken from lines along posterior cortices of distal femur and proximal tibia. Normal variability in distal femoral bowing [22] may therefore have contributed to the greater variability of measures between their DPG and criterion measurements. Our results also compare favourably to those of Verhaegen et al. (2010) particularly in knee flexion. Their range of LOA whilst not explicitly stated in their results can be interpreted from the Bland-Altman scatter plot to be approximately $23^{\circ}$ in flexion and $11^{\circ}$ in extension. Neither of these studies assessed subjects who had undergone TKA; Naylor et al. (2011) used normal and pre-operative TKA subjects and Verhaegen et al. (2010) used the nonsymptomatic knees of orthopaedic patients. Our LOA were however wider than those reported by Russell et al. (2003) who evaluated an internet-based camera. This study evaluated the knee ROM of one young, healthy subject in a non-clinical, laboratory environment and the range in our TKA population in variables such as BMI (19.9 - 46) may have contributed to this discrepancy.

Intra-tester reliability was found to be slightly higher than inter-tester reliability which is consistent with other reports in the literature $[8,14,15,23]$, however all ICC's were greater than 0.9 indicating excellent (almost perfect) correlation. Furthermore the mean differences and standard deviations for intra and inter-rater measurements were very small (all $<1.4^{\circ}$ ) suggesting different clinicians can use this technique interchangeably. Studies of conventional goniometry [3] and digital photography $[6,12,14]$ have found measurement of knee extension to be less reliable than flexion. Our ICC results demonstrate a similar trend, though both inter and intra-tester ICC's were excellent and mean and mean absolute differences were less than two degrees. Our high agreement in knee extension measures which are comparable to those reported in studies using populations with arthritic knees [6] may reflect a higher variability in extension ROM $\left(5.5^{\circ}\right.$ hyperextension to fixed flexion contracture of $20^{\circ}$ ) compared to the healthy knees used in other studies [7,14].

This study has demonstrated that with minimal training the DPG in the hands of experienced clinicians is a reliable and valid tool for measurement of knee ROM at TKA review follow-up. It could therefore be a valuable tool in clinical settings as it has several advantages over the UG. First and foremost is versatility and accessibility. Photographs can be taken from an increasing number of digital devices including mobile phones and tablet devices and analysis software can be installed on these devices to provide instant measurement. This study demonstrated that provided the camera is visually aligned with the knee axis of rotation more rigorous protocols such as measuring distances and using tripods are not required. Moreover images can be stored, assessed, and re-assessed by any number of clinicians reducing the risk of transcription and measurement error. This would be useful for surgeons and clinicians to ascertain patient progression but also has potential for use in research particularly in studies evaluating the effects of prosthetic innovations or surgical interventions in improving knee ROM.

The visual estimation of the knee axis has been described as the "Achilles heel" of goniometry $[9,10]$ however localising the greater trochanter, even with pal- 
pation for UG measurement, is potentially more problematic as it is impeded by adiposity [12]. The technique described in this study minimises the risk of landmark placement error by allowing the assessors to adjust landmark and axis placement after their initial placement until they are satisfied the adjoining lines correspond to the lines of femur and fibula. Moreover, the DPG allows direct placement of landmark points at the greater trochanter and lateral malleolus, beyond the reach of a standard UG on an adult lower limb, and this may improve accuracy similar to that when using a long arm UG [24].

A limitation of this study is that only one photograph was taken and all measures were performed from that image. We acknowledge the possibility that any error with this measurement technique may arise from inaccuracy in the taking of the photograph, however our photographic protocol followed guidelines reported in the literature which demonstrated that if the photographer takes the image within $5^{\circ}$ of the angle of incident, error will be minimal [13]. Sample size was not calculated a priori but involved all available participants in a TKR study. Though validity was excellent and standard deviations small, a larger sample may have provided more robust data. A final limitation of this study was the use of a single assessor for validity and only two assessors for reliability. Future research using assessors with a range of experience is recommended to improve the generalizability of the results.

\section{Acknowledgements}

The authors wish to gratefully acknowledge the assistance of Paul Miller who assisted with data collection.

\section{REFERENCES}

[1] M. A. Ritter, J. D. Lutgring, K. E. Davis and M. E. Berend, "The Effect of Postoperative Range of Motion on Functional Activities after Posterior Cruciate-Retaining Total Knee Arthroplasty," Journal of Bone and Joint Surgery, (American), Vol. 90, No. 4, 2008, pp. 777-784.

[2] M. S. Austin, E. Ghanem, A. Joshi, R. Trappler, J. Parvizi, and W. J. Hozack, "The Assessment of Intraoperative Prosthetic Knee Range of Motion using Two Methods," Journal of Arthroplasty, Vol 23, No. 4, 2008, pp. 515-521. doi:10.1016/j.arth.2007.12.011

[3] C. Lavernia, M. D’Apuzzo, M. D. Rossi and D. Lee, “Accuracy of Knee Range of Motion Assessment after Total Knee Arthroplasty," Journal of Arthroplasty, Vol. 23, No. 6, 2008, pp. 85-91. doi:10.1016/j.arth.2008.05.019

[4] P. P. Gogia, J. H. Braatz, S. J. Rose and B. J. Norton, "Reliability and Validity of Goniometric Measurements at the Knee," Physical Therapy, Vol. 67, No. 2, 1987, pp. 192-195.
[5] J. Z. Edwards, K. A. Greene, R. S. Davis, M. W. Kovacik, D. A. Noe and M. J. Askew, "Measuring Flexion in Knee Arthroplasty Patients," Journal of Arthroplasty, Vol. 19, No. 3, 2004, pp. 69-72. doi:10.1016/j.arth.2003.12.001

[6] D. Bennett, B. Hanratty, N. Thompson and D. Beverland, "Measurement Of Knee Joint Motion Using Digital Imaging," International Orthopaedics, Vol. 33, No. 6, 2009 , pp. 1627-1631. doi:10.1007/s00264-008-0694-9

[7] P. Piriyaprasarth and M. E. Morris, "Psychometric Properties of Measurement Tools for Quantifying Knee Joint Position and Movement: A Systematic Review," Knee, Vol. 14, No. 1, 2007, pp. 2-8.

doi:10.1016/j.knee.2006.10.006

[8] T. Russell, G. Jull and R. Wootton, "Can the Internet Be Used as a Medium to Evaluate Knee Angle?" Manual Therapy, Vol. 8, No. 4, 2003, pp. 242-246. doi:10.1016/S1356-689X(03)00016-X

[9] L. Brosseau, S. Balmer, M. Tousignant J. P. O'Sullivan, C. Goudreault, M. Goudreault and S. Gringas, "Intra- and Intertester Reliability and Criterion Validity of the $\mathrm{Pa}$ rallelogram and Universal Goniometers for Measuring Maximum Active Knee Flexion and Extension of Patients with Knee Restrictions," Archives of Physical Medicine and Rehabilitaion, Vol. 82, No. 3, 2001, pp. 396-402. doi:10.1053/apmr.2001.19250

[10] L. Brosseau, M. Tousignant, J. Budd, N. Chartier, L. Duciaume, S. Plamondon, J. P. O'Sullivan, S. O'Donoghue and S. Balmer, "Intratester and Intertester Reliability and Criterion Validity of the Parallelogram and Universal Goniometers for Active Knee Flexion in Healthy Subjects," Physiotherapy Research International, Vol. 2, No. 3, 1997, pp. 150-166. doi:10.1002/pri.97

[11] A. F. Lenssen, E. M. van Dam, Y. H. Crijns, M. Verhey, R. J. Geesink, P. A. van den Brandt and R. A. de Bie, "Reproducibility of Goniometric Measurement of the Knee in the In-Hospital Phase Following Total Knee Arthroplasty," BMC Musculoskeletal Disorders, Vol. 8, No. 83, 2007.

[12] J. M. Naylor, V. Ko, S. Adie, C. Gaskin, R. Walker, I. A. Harris and R. Mittal, "Validity and Reliability of Using Photography for Measuring Knee Range of Motion: A Methodological Study," BMC Musculoskelet Disorders, Vol. 12, No. 77, 2011.

[13] C. Dunlevy, M. Cooney and J. Gormely, "Procedural Considerations for Photographic-Based Joint Angle Measurements," Physiotherapy Research International, Vol. 10, No. 4, 2005, pp. 190-200. doi:10.1002/pri.9

[14] F. Verhaegen, Y. Ganseman, N. Arnout, H. Vandenneucker and J. Bellemans, "Are Clinical Photographs Appropriate to Determine the Maximal Range of Motion of the Knee?" Acta Orthopedica Belgica, Vol. 76, No. 6, 2010, pp. 794-798.

[15] E. Karkouti and R. Marks, "Reliability of Photographic Range of Motion Measurements in a Healthy Sample: Knee and Ankle Joint Measurment," Physiotherapy Canada, Vol. 49, 1997, pp. 24-31.

[16] D. C. Lee, D. H. Kim, R. D. Scott and K. Suthers, "Intraoperative Flexion Against Gravity as an Indication of Ul- 
timate Range of Motion in Individual Cases after Total Knee Arthroplasty," Journal of Arthroplasty, Vol. 13, No. 5, 1998, pp. 500-503. doi:10.1016/S0883-5403(98)90047-X

[17] R. W. Nutton, M. L. van der Linden, P. J. Rowe, P. Gaston and F. A. Wade, "A Prospective Randomised Double-Blind Study of Functional Outcome and Range of Flexion Following Total Knee Replacement with the Nexgen Standard and High Flexion Components," Journal of Bone and Joint Surgery, (British) Vol. 90, No. 1, 2008, pp. 37-42.

[18] C. Norkin and D. White, "Measurement of Joint Motion: A Guide to Goniometry," 2nd Edition, F. A. Davis Company, Philadelphia, 1995.

[19] J. M. Bland and D. G. Altman, "Statistical Methods for Assessing Agreement Between Two Methods of Clinical Measurement," Lancet, Vol. 1, No. 8476, 1986, pp. 307310. doi:10.1016/S0140-6736(86)90837-8
[20] J. M. Bland and D. G. Altman, "Measuring Agreement In Method Comparison Studies," Statistical Methods in Medical Research, Vol. 8, No. 2, 1999, pp. 135-160. doi:10.1191/096228099673819272

[21] J. R. Landis and G. G. Koch, "The Measurement of Observer Agreement for Categorical Data," Biometrics, Vol. 33, No. 1, 1977, pp. 159-174. doi:10.2307/2529310

[22] T. M. Yehyawi, J. J. Callaghan, D. R. Pedersen, M. R. O'Rourke and S. S. Liu, "Variances in Sagittal Femoral Shaft Bowing in Patients Undergoing TKA," Clinical Orthopaedics and Related Research, Vol. 464, No. 1, 2007, pp. 99-104.

[23] D. C. Boone, S. P. Azen, C. M. Lin, C. Spence, C. Baron and L. Lee, "Reliability of Goniometric Measurements," Physical Therapy, Vol. 58, No. 11, 1978, pp. 1355-1390.

[24] H. M. Clarkson and G. Gilewich, "Musculoskeletal Assessment: Joint Range of Motion and Manual Muscle Strength," Williams \& Wilkins, Baltimore, 1989. 